\title{
Common Fixed Point Theorem for Compatible Maps of Type $(\beta)$ and Type $(\alpha)$ using Integral Type Mapping
}

\author{
M. Ramana Reddy, PhD \\ Associate Professor of Mathematics \\ Sreenidhi Institute of Science and Technology, Hyderabad
}

\begin{abstract}
In this paper we prove a common fixed point theorem for compatible map of type $(\beta)$ in Fuzzy 2- metric space.
\end{abstract}

\section{Keywords}

Fuzzy 2- metric space, G- Cauchy sequence, Weakly compatible, point of coincidence, complete metric space

\section{INTRODUCTION}

Integral type contraction principle is one of the most popular contraction principle in fixed point theory. The first known result in this direction was given by Branciari [1] in general setting of lebgesgue integrable function and proved following fixed point theorems in metric spaces. In 1988, Grabiec [3] defined contraction and contractive mappings on a fuzzy metric space and extended fixed point theorems of Banach and Edelstein in such spaces. Following Grabiec's approach, Mishra et al. [4] obtained common fixed point theorems for asymptotically commuting mappings on fuzzy metric spaces. In 1998, Vasuki [5] established a generalization of Grabiec's fuzzy contraction theorem wherein he proved a common fixed point theorem for a sequence of mappings in a fuzzy metric space. Thereafter, Cho [2] extended the concept of compatible mappings of type (alpha)

Our objective of this paper is to prove a common fixed point theorem by removing the assumption of continuity, relaxing compatibility to compatible maps of type $(\alpha)$ or $(\beta)$. weak compatibility and replacing the completeness of the space with a set of alternative conditions for functions satisfying an implicit relation in FM-space.

In this paper the following implicit relation: Let $\mathrm{I}=[0,1]$,* be a continuous t-norm and $\mathrm{F}$ be the set of all real continuous functions $\mathrm{F}: \mathrm{I}^{6} \rightarrow \mathrm{R}$ satisfying the following conditions

1.1 $\mathrm{F}$ is no increasing in the fifth and sixth variables,

1.2 if, for some constant $\mathrm{k} \in(0,1)$ we have

$$
\text { 1.2(a) } \mathrm{F}\left(\mathrm{u}(\mathrm{kt}), \mathrm{v}(\mathrm{t}), \mathrm{v}(\mathrm{t}), \mathrm{u}(\mathrm{t}), 1, \mathrm{u}\left(\frac{\mathrm{t}}{2}\right) * \mathrm{v}\left(\frac{\mathrm{t}}{2}\right)\right) \geq 1 \text {, }
$$

$$
\text { or }
$$

1.2(b) F (u(kt), v(t), u(t), v(t), u( $\left.\left.\frac{\mathrm{t}}{2}\right) * \mathrm{v}\left(\frac{\mathrm{t}}{2}\right), 1\right) \geq 1$

for any fixed $\mathrm{t}>0$ and any nondecreasing functions $\mathrm{u}, \mathrm{v}:(0, \infty) \rightarrow$ I with $0 \leq \mathrm{u}(\mathrm{t}), \mathrm{v}(\mathrm{t}) \leq 1$ then there exists $\mathrm{h} \in(0,1)$ with $\mathrm{u}(\mathrm{ht}) \geq \mathrm{v}(\mathrm{t}) * \mathrm{u}(\mathrm{t})$,

1.3 if, for some constant $\mathrm{k} \in(0,1)$ we have $\mathrm{F}(\mathrm{u}(\mathrm{kt}), \mathrm{u}(\mathrm{t}), 1,1, \mathrm{u}(\mathrm{t}), \mathrm{u}(\mathrm{t})) \geq 1$

for any fixed $\mathrm{t}>0$ and any nondecreasing function $\mathrm{u}$ : $(0, \infty) \rightarrow$ I then $\mathrm{u}(\mathrm{kt}) \geq \mathrm{u}(\mathrm{t})$

\section{PRELIMINARIES}

Definition - 2.1 A triplet $(X, M, \star)$ is said to be a Fuzzy 2metric space if $X$ is an arbitrary set, $\star$ is a continuous $t-$ norm and $M$ is a fuzzy set on $X^{2} \times(0, \infty)$ satisfying the following condition for all $\mathrm{x}, \mathrm{y}, \mathrm{z}, \mathrm{s}, \mathrm{t}>0$

$(\mathrm{FM}-1) \mathrm{M}(\mathrm{x}, \mathrm{y}, \theta, \mathrm{t})>0$

$(F M-2) M(x, y, \theta, t)=1$ if and only if $x=y=\theta$.

$(F M-3) M(x, y, \theta, t)=M(y, \theta, x, t)=M(\theta, x, y, t)$

$(F M-4) \quad M(x, y, \theta, t) \star M(y, z, \theta, s) \star M(z, x, \theta, q) \leq$ $M(x, y, z, t+s+q)$

$(\mathrm{FM}-5) \mathrm{M}(\mathrm{x}, \mathrm{y}, \theta, \bullet):(0, \infty) \rightarrow(0,1]$ is continuous.

Then $M$ is called a Fuzzy 2- metric on $X$. The function $M(x, y, \theta, t)$ denote the degree of nearness between $x, y$ and $\theta$ with respect to $t$.

Example : Let $(\mathrm{X}, \mathrm{d})$ be a metric space. Define $\mathrm{a} * \mathrm{~b}=$ $\min \{a, b\}$ and $M(x, y, \theta, t)=\frac{t}{t+d(x, y, \theta)}$

For all $x, y \in X$ and all $t>0$. Then $(X, M, \star)$ is a Fuzzy 2metric space.

It is called the Fuzzy 2- metric space induced by d.We note that, $M(x, y, \theta, t)$ can be realized as the measure of nearness between $\mathrm{x}$ and $\mathrm{y}$ with respect to $\mathrm{t}$. It is known that $M(x, y, \cdot)$ is non decreasing for all $x, y \in X$. Let $M(x, y, \star)$ be a Fuzzy 2- metric space for $t>0$, the open ball $\mathrm{B}(\mathrm{x}, \mathrm{r}, \theta, \mathrm{t})=\{\mathrm{y} \in \mathrm{X}: \mathrm{M}(\mathrm{x}, \mathrm{y}, \theta, \mathrm{t})>1-\mathrm{r}\}$.

Now, the collection $\{B(x, r, \theta, t): x \in X, 0<r<1, t>0\}$ is a neighborhood system for a topology $\tau$ on $\mathrm{X}$ induced by the Fuzzy 2- metric M. This topology is Housdroff and first countable.

Definition 2.2 A sequence $\left\{x_{n}\right\}$ in a Fuzzy 2- metric space $(X, M, \star)$ is said to be a converges to $x$ iff for each $\varepsilon>0$ and each $t>0, n_{0} \in N$ such that $M\left(x_{n}, x, \theta, t\right)>1-\varepsilon$ for all $n \geq n_{0}$.

Definition 2.3 A sequence $\left\{x_{n}\right\}$ in a Fuzzy 2- metric space $(\mathrm{X}, \mathrm{M}, \star)$ is said to be a $\mathrm{G}$ - Cauchy sequence converges to $x$ iff for each $\varepsilon>0$ and each $t>0, n_{0} \in N$ such that $M\left(x_{m}, x_{n}, \theta, t\right)>1-\varepsilon$ for all $m, n \geq n_{0}$.

A Fuzzy 2- metric space $(X, M, \star)$ is said to be complete if every G- Cauchy sequence in it converges to a point in it.

\section{MAIN RESULT}

Theorem 3.1 Let $(X, M, \star)$ be a complete Fuzzy 2- metric space and let $A, B, S, T, P$ and $Q$ be mappings from $X$ into itself such that the following conditions are satisfied:

3.1(a) $\mathrm{P}(\mathrm{X}) \subset \mathrm{ST}(\mathrm{X})$ and $\mathrm{Q}(\mathrm{X}) \subset \mathrm{AB}(\mathrm{X})$, 
3.1 (b) $(\mathrm{P}, \mathrm{AB})$ is compatible of type $(\beta)$ and $(\mathrm{Q}, \mathrm{ST})$ is weak compatible,

3.1(c) there exists $k \in(0,1)$ such that for every $x, y \in$ $\mathrm{X}$ and $\mathrm{t}>0$

$\mathrm{F}\left(\mathrm{M}^{2}(\mathrm{Px}, \mathrm{Qy}, \theta, \mathrm{kt}), \mathrm{M}^{2}(\mathrm{ABx}, \mathrm{STy}, \theta, \mathrm{t}), \mathrm{M}^{2}(\mathrm{Px}, \mathrm{ABx}, \theta, \mathrm{t})\right.$,

$\int_{0}^{F}\left(\begin{array}{c}M^{2}(P y, S T y, \theta, t), M^{2}(P x, S T y, \theta, t), M^{2}(A B x, Q y, \theta, t) \\ M^{2}\left(P^{2}\right)\end{array}\right) d v \geq 1$

Where $\xi:[0,+\infty] \rightarrow[0,+\infty]$ is a lebgesgue integrable mapping which is summable on each compact subset of $[0,+\infty]$ non negative and such that $\forall \varepsilon>0, \int_{0}^{\varepsilon} \xi(\mathrm{v}) \mathrm{dv}>$ 0 . Then A, B,S,T, $P$ and $Q$ have a unique common fixed point in $\mathrm{X}$.

Proof : Let $\mathrm{x}_{0} \in \mathrm{X}$, then from 3.1 (a) we have $\mathrm{x}_{1}, \mathrm{x}_{2} \in \mathrm{X}$ such tha $\mathrm{Px}_{0}=\mathrm{STx}_{1}$ and $\mathrm{Qx}_{1}=\mathrm{ABx}_{2}$

Inductively, we construct sequences $\left\{x_{n}\right\}$ and $\left\{y_{n}\right\}$ in $X$ such that for $\mathrm{n} \in \mathrm{N}$

$\mathrm{Px}_{2 \mathrm{n}-2}=\operatorname{STx}_{2 \mathrm{n}-1}=\mathrm{y}_{2 \mathrm{n}-1}$ and $\mathrm{Qx}_{2 \mathrm{n}-1}=\mathrm{ABx}_{2 \mathrm{n}}=\mathrm{y}_{2 \mathrm{n}}$ putx $=x_{2 n}$ and $y=x_{2 n+1}$ in 3.1(c)then we have

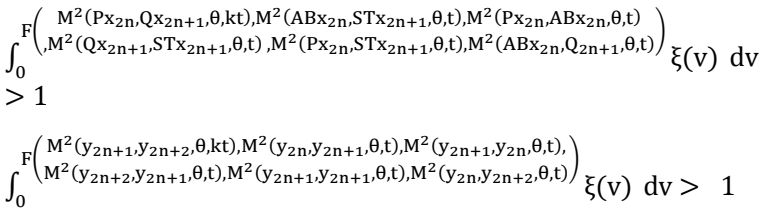

From condition 3.1 (a) we have

$\int_{0}^{M^{2}\left(y_{2 n+1}, y_{2 n+2}, \theta, k t\right)} \xi(v) d v \geq$

$\int_{0}^{M^{2}\left(y_{2 n}, y_{2 n+1}, \theta, \frac{\mathrm{t}}{2}\right) \star M^{2}\left(y_{2 n+2}, y_{2 n+1}, \theta, \frac{t}{2}\right)} \xi(v) d v$

we have

$\int_{0}^{M^{2}\left(y_{2 n+1}, y_{2 n+2}, \theta, k t\right)} \xi(v) d v \geq \int_{0}^{M^{2}\left(y_{2 n}, y_{2 n+1}, \theta, \frac{t}{2}\right)} \xi(v) d v$

Since $\xi(v)$ is a lebesgue integrable function so we have

$M\left(y_{2 n+1}, y_{2 n+2}, \theta, k t\right) \geq M\left(y_{2 n}, y_{2 n+1}, \theta, \frac{t}{2}\right)$

Similarly we have

$M\left(y_{2 n+2}, y_{2 n+3}, \theta, k t\right) \geq M\left(y_{2 n+1}, y_{2 n+2}, \theta, \frac{t}{2}\right)$

Thus we have

$M\left(y_{n+1}, y_{n+2}, \theta, k t\right) \geq M\left(y_{n}, y_{n+1}, \theta, \frac{t}{2}\right)$

$M\left(y_{n+1}, y_{n+2}, \theta, t\right) \geq M\left(y_{n}, y_{n+1}, \theta, \frac{t}{2^{k}}\right)$

$M\left(y_{n}, y_{n+1}, \theta, t\right) \geq M\left(y_{0}, y_{1}, \theta, \frac{t}{2^{n k}}\right) \rightarrow 1$ as $n \rightarrow \infty$,

and hence $\mathrm{M}\left(\mathrm{y}_{\mathrm{n}}, \mathrm{y}_{\mathrm{n}+1}, \theta, \mathrm{t}\right) \rightarrow 1$ as $\mathrm{n} \rightarrow \infty$ for all $\mathrm{t}>0$.

For each $\epsilon>0$ and $t>0$, we can choose $\mathrm{n}_{0} \in \mathrm{N}$ such that $M\left(y_{n}, y_{n+1}, \theta, t\right)>1-\epsilon$ for all $n>n_{0}$.

For any $m, n \in N$ we suppose that $m \geq n$. Then we have

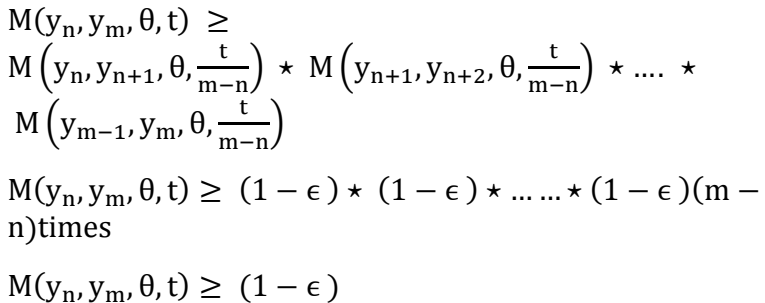

And hence $\left\{y_{n}\right\}$ is a Cauchy sequence in $X$.

Since $(X, M, \star)$ is complete, $\left\{y_{n}\right\}$ converges to some point $\mathrm{z} \in \mathrm{X}$. Also its subsequences converges to the same point $\mathrm{z} \in \mathrm{X}$.

That is $\left\{\mathrm{Px}_{2 \mathrm{n}+2}\right\} \rightarrow \mathrm{z}$ and $\left\{\mathrm{STx}_{2 \mathrm{n}+1}\right\} \rightarrow \mathrm{z}$

$$
\left\{\mathrm{Qx}_{2 \mathrm{n}+1}\right\} \rightarrow \mathrm{z} \text { and }\left\{\mathrm{ABx}_{2 \mathrm{n}}\right\} \rightarrow \mathrm{z}
$$

As $(P, A B)$ is compatible pair of type $(\beta)$, we have

$\mathrm{M}\left(\mathrm{PPx}_{2 \mathrm{n}},(\mathrm{AB})(\mathrm{AB}) \mathrm{x}_{2 \mathrm{n}}, \theta, \mathrm{t}\right)=1$, for all $\mathrm{t}>0$

Or $\mathrm{M}\left(\mathrm{PPx}_{2 n}, \mathrm{ABz}, \theta, \mathrm{t}\right)=1$ Therefore, $\mathrm{PPx}_{2 \mathrm{n}} \rightarrow \mathrm{ABz}$.

Put $\quad \mathrm{x}=(\mathrm{AB}) \mathrm{x}_{2 \mathrm{n}}$ and $\mathrm{y}=\mathrm{x}_{2 \mathrm{n}+1}$ in 3.1(c)we have $M^{2}\left(P(A B) x_{2 n}, Q y, \theta, k t\right), M^{2}\left(A B(A B) x_{2 n}, S T x_{2 n+1}, \theta, t\right)$

$\mathrm{F}\left(\mathrm{M}^{2}\left(\mathrm{P}(\mathrm{AB}) \mathrm{x}_{2 \mathrm{n}}, \mathrm{AB}(\mathrm{AB}) \mathrm{x}_{2 \mathrm{n}}, \theta, \mathrm{t}\right), \mathrm{M}^{2}\left(\mathrm{Qx}_{2 \mathrm{n}+1}, \mathrm{STx}_{2 \mathrm{n}+1}, \theta, \mathrm{t}\right)\right.$,

$\left.\int_{0} M^{2}\left(\mathrm{P}(\mathrm{AB}) \mathrm{x}_{2 n}, S T x_{2 n+1}, \theta, t\right), M^{2}\left(\mathrm{AB}(\mathrm{AB}) \mathrm{x}_{2 \mathrm{n}}, \mathrm{Qx} \mathrm{x}_{2 \mathrm{n}+1}, \theta, \mathrm{t}\right)\right) \xi(\mathrm{v}) \mathrm{dv}>1$

Taking $\mathrm{n} \rightarrow \infty$ and 3.1(a) we get

$\int_{0}^{M^{2}((A B) z, z, \theta, k t)} \xi(v) d v \geq \int_{0}^{M^{2}((A B) z, z, \theta, t)} \xi(v) d v$

Since $\xi(v)$ is a lebesgue integrable function which implies

$$
\mathrm{M}((\mathrm{AB}) \mathrm{z}, \mathrm{z}, \theta, \mathrm{kt}) \geq \mathrm{M}((\mathrm{AB}) \mathrm{z}, \mathrm{z}, \theta, \mathrm{t})
$$

we have $\quad \mathrm{ABz}=\mathrm{z}$

Put $\mathrm{x}=\mathrm{z}$ and $\mathrm{y}=\mathrm{x}_{2 \mathrm{n}+1}$ in 3.1(c) we have

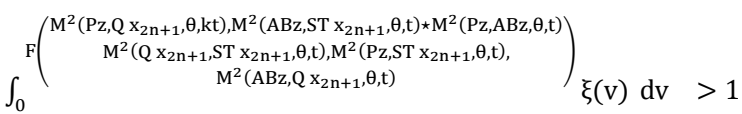

Taking $\mathrm{n} \rightarrow \infty 3.1$ (a) That is $\int_{0}^{\mathrm{M}^{2}(\mathrm{Pz}, \mathrm{z}, \theta, \mathrm{kt})} \xi(\mathrm{v}) \mathrm{dv} \geq$ $\int_{0}^{\mathrm{M}^{2}(\mathrm{Pz}, \mathrm{z}, \theta, \mathrm{t})} \xi(\mathrm{v}) \mathrm{dv}$

Since $\xi(v)$ is a lebesgue integrable function so we have

$\mathrm{M}(\mathrm{Pz}, \mathrm{z}, \theta, \mathrm{kt}) \geq \mathrm{M}(\mathrm{Pz}, \mathrm{z}, \theta, \mathrm{t})$

we get $\mathrm{Pz}=\mathrm{z}$

So we have $\mathrm{ABz}=\mathrm{Pz}=\mathrm{z}$.

Putting $\mathrm{x}=\mathrm{Bz}$ and $\mathrm{y}=\mathrm{x}_{2 \mathrm{n}+1}$ in 3.1(c), we get

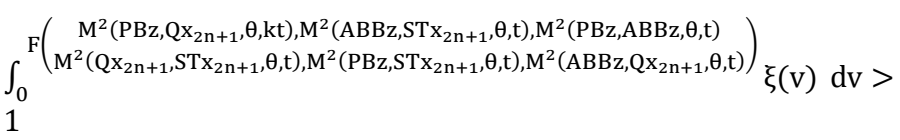

Taking $\mathrm{n} \rightarrow \infty, 3.1(\mathrm{a})$

$\int_{0}^{\mathrm{M}^{2}(\mathrm{Bz}, \mathrm{z}, \theta \mathrm{kt})} \xi(\mathrm{v}) \mathrm{dv} \geq \int_{0}^{\mathrm{M}^{2}(\mathrm{Bz}, \mathrm{z}, \theta, \mathrm{t})} \xi(\mathrm{v}) \mathrm{dv}$

Since $\xi(v)$ is a lebesgue integrable function which follows

$\mathrm{M}(\mathrm{Bz}, \mathrm{z}, \theta, \mathrm{kt}) \geq \mathrm{M}(\mathrm{Bz}, \mathrm{z}, \theta, \mathrm{t})$

we have $\mathrm{Bz}=\mathrm{z}$

And also we have $\mathrm{ABz}=\mathrm{z}$ implies $\mathrm{Az}=\mathrm{z}$ 
Therefore $\mathrm{Az}=\mathrm{Bz}=\mathrm{Pz}=\mathrm{z}$.

As $\mathrm{P}(\mathrm{X}) \subset \mathrm{ST}(\mathrm{X})$ there exists $\mathrm{u} \in \mathrm{X}$ such that

$\mathrm{z}=\mathrm{Pz}=\mathrm{STu}$

Putting $\quad \mathrm{x}=\mathrm{x}_{2 \mathrm{n}}$ and $\mathrm{y}=\mathrm{u} \quad$ in $3.1(\mathrm{c}) \quad$ we get

$\mathrm{F}\left(\mathrm{M}^{2}\left(\mathrm{Px}_{2 n}, \mathrm{Qu}, \theta, \mathrm{kt}\right), \mathrm{M}^{2}\left(\mathrm{ABx}_{2 n}, \mathrm{STu}, \theta, \mathrm{t}\right), \mathrm{M}^{2}\left(\mathrm{Px}_{2 \mathrm{n}}, \mathrm{ABx} \mathrm{x}_{2 n}, \theta, \mathrm{t}\right)\right)$

$\int_{0}\left(\mathrm{M}^{2}(\mathrm{Qu}, \mathrm{STu}, \theta, \mathrm{t}), \mathrm{M}^{2}\left(\mathrm{Px}_{2 \mathrm{n}}, \mathrm{STu}, \theta, \mathrm{t}\right), \mathrm{M}^{2}\left(\mathrm{ABx}_{2 \mathrm{n}}, \mathrm{Qu}, \theta, \mathrm{t}\right) \quad \xi(\mathrm{v}) \mathrm{dv}>\right.$

1

Taking $\mathrm{n} \rightarrow \infty$ we get

$F\left(M^{2}(z, Q u, \theta, k t), M^{2}(z, S T u, \theta, t), M^{2}(z, z, \theta, t)\right.$

$\int_{0}^{F\left(M^{2}(\mathrm{Qu}, \mathrm{STu}, \theta, \mathrm{t}), \mathrm{M}^{2}(\mathrm{z}, \mathrm{STu}, \theta, \mathrm{t}), \mathrm{M}^{2}(\mathrm{z}, \mathrm{Qu}, \theta, \mathrm{t})\right.} \xi(\mathrm{v}) \mathrm{dv}>1$

$\int_{0}^{M^{2}(z, Q u, \theta, k t)} \xi(v) d v \geq \int_{0}^{M^{2}(z, Q u, \theta, t)} \xi(v) d v$

Since $\xi(v)$ is a lebesgue integrable function which implies

$\mathrm{M}(\mathrm{z}, \mathrm{Qu}, \theta, \mathrm{kt}) \geq \mathrm{M}(\mathrm{z}, \mathrm{Qu}, \theta, \mathrm{t})$

we have $\mathrm{Qu}=\mathrm{z}$

Hence $\mathrm{STu}=\mathrm{z}=\mathrm{Qu}$.

Hence $(\mathrm{Q}, \mathrm{ST})$ is weak compatible, therefore, we have

$\mathrm{QSTu}=\mathrm{STQu}$

Thus $\mathrm{Qz}=\mathrm{STz}$.

Putting $\mathrm{x}=\mathrm{x}_{2 \mathrm{n}}$ and $\mathrm{y}=\mathrm{z}$ in $3.1 \odot$ we get

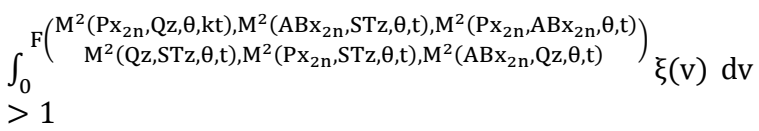

Taking $\mathrm{n} \rightarrow \infty$ we get

$F\left(M^{2}(z, Q z, \theta, k t), M^{2}(z, S T z, \theta, t), M^{2}(z, z, \theta, t)\right.$

$\int_{0}^{F\left(M^{2}(Q z, S T z, \theta, t), M^{2}(z, S T z, \theta, t), M^{2}(z, Q z, \theta, t)\right.} \xi(v) d v>1$

$\int_{0}^{\mathrm{M}^{2}(\mathrm{z}, \mathrm{Qz}, \theta, \mathrm{kt})} \xi(\mathrm{v}) \mathrm{dv} \geq \int_{0}^{\mathrm{M}^{2}(\mathrm{z}, \mathrm{Qz}, \theta, \mathrm{t})} \xi(\mathrm{v}) \mathrm{dv}$

Since $\xi(v)$ is a lebesgue integrable function and hence

$\mathrm{M}(\mathrm{z}, \mathrm{Qz}, \theta, \mathrm{kt}) \geq \mathrm{M}(\mathrm{z}, \mathrm{Qz}, \theta, \mathrm{t})$

we get $\mathrm{Qz}=\mathrm{z}$.

Putting $\mathrm{x}=\mathrm{x}_{2 \mathrm{n}}$ and $\mathrm{y}=\mathrm{Tz}$ in 5.3.2(c) we get

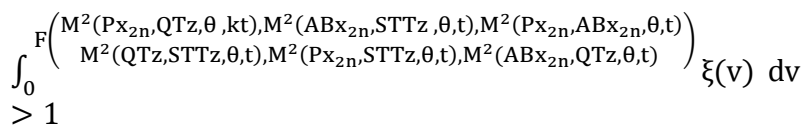

As $\mathrm{QT}=\mathrm{TQ}$ and $\mathrm{ST}=\mathrm{TS}$ we have

$\mathrm{QTz}=\mathrm{TQz}=\mathrm{Tz}$

And $\mathrm{ST}(\mathrm{Tz})=\mathrm{T}(\mathrm{STz})=\mathrm{TQz}=\mathrm{Tz}$.

Taking $\mathrm{n} \rightarrow \infty$ we get

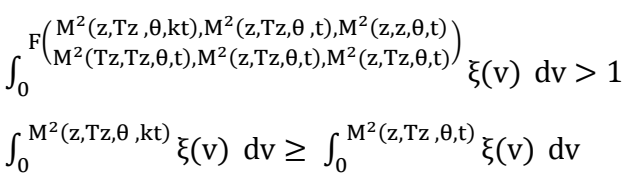

Since $\xi(v)$ is a lebesgue integrable function therefore

$\mathrm{M}(\mathrm{z}, \mathrm{Tz}, \theta, \mathrm{kt}) \geq \mathrm{M}(\mathrm{z}, \mathrm{Tz}, \theta, \mathrm{t})$

we have $\mathrm{Tz}=\mathrm{z}$
Now $\mathrm{STz}=\mathrm{Tz}=\mathrm{z}$ implies $\mathrm{Sz}=\mathrm{z}$.

Hence $\quad \mathrm{Sz}=\mathrm{Tz}=\mathrm{Qz}=\mathrm{z}$

Combining $\quad \mathrm{Az}=\mathrm{Bz}=\mathrm{Pz}=\mathrm{Sz}=\mathrm{Tz}=\mathrm{Qz}=\mathrm{z}$

Hence $\mathrm{z}$ is the common fixed point of $\mathrm{A}, \mathrm{B}, \mathrm{S}, \mathrm{T}, \mathrm{P}$ and $\mathrm{Q}$.

Uniqueness Let $\mathrm{u}$ be another common fixed point of $\mathrm{A}, \mathrm{B}, \mathrm{S}, \mathrm{T}, \mathrm{P}$ and $\mathrm{Q}$. Then $\mathrm{Au}=\mathrm{Bu}=\mathrm{Su}=\mathrm{Tu}=\mathrm{Pu}=\mathrm{Qu}=$ $\mathrm{u}$ Putting $\mathrm{x}=\mathrm{u}$ and $\mathrm{y}=\mathrm{z}$ in 3.1(c) then we get $\int_{0}^{F\left(\begin{array}{c}M^{2}(\mathrm{Pu}, \mathrm{Qz}, \theta, \mathrm{kt}), \mathrm{M}^{2}(\mathrm{ABu}, \mathrm{STz}, \theta, \mathrm{t}), \mathrm{M}^{2}(\mathrm{Pu}, \mathrm{ABu}, \theta, \mathrm{t}) \\ \mathrm{M}^{2}(\mathrm{Qz}, \mathrm{STz}, \theta, \mathrm{t}), \mathrm{M}^{2}(\mathrm{Pu}, \mathrm{STz}, \theta, \mathrm{t}), \mathrm{M}^{2}(\mathrm{ABu}, \mathrm{Qz}, \theta, \mathrm{t})\end{array}\right)} \xi(\mathrm{v}) \mathrm{dv}>1$

Taking limit both side then we get

$\int_{0}^{F\left(\begin{array}{c}M^{2}(u, z, \theta, k t), M^{2}(u, z, \theta, t), M^{2}(u, u, \theta, t) \\ M^{2}(z, z, \theta, t), M^{2}(u, z, \theta, t), M^{2}(u, z, \theta, t)\end{array}\right)} \xi(v) d v>1$

$\int_{0}^{\mathrm{M}^{2}(\mathrm{u}, \mathrm{z}, \theta, \mathrm{kt})} \xi(\mathrm{v}) \mathrm{dv} \geq \int_{0}^{\mathrm{M}^{2}(\mathrm{u}, \mathrm{z}, \theta, \mathrm{t})} \xi(\mathrm{v}) \mathrm{dv}$

Since $\xi(v)$ is a lebesgue integrable function so we have

$\mathrm{M}(\mathrm{u}, \mathrm{z}, \theta, \mathrm{kt}) \geq \mathrm{M}(\mathrm{u}, \mathrm{z}, \theta, \mathrm{t})$

we get $\mathrm{z}=\mathrm{u}$.

That is $\mathrm{z}$ is a unique common fixed point of $\mathrm{A}, \mathrm{B}, \mathrm{S}, \mathrm{T}, \mathrm{P}$ and $\mathrm{Q}$ in $\mathrm{X}$.

Corollary 3.2 Let $(X, M, \star)$ be a complete Fuzzy 2- metric space and let $A, S, P$ and $Q$ be mappings from $X$ into itself such that the following conditions are satisfied:

(a) $\mathrm{P}(\mathrm{X}) \subset \mathrm{S}(\mathrm{X})$ and $\mathrm{Q}(\mathrm{X}) \subset \mathrm{A}(\mathrm{X})$,

(b) $(\mathrm{P}, \mathrm{A})$ is compatible of type $(\beta)$ and $(\mathrm{Q}, \mathrm{S})$ is weak compatible,

(c) there exists $\mathrm{k} \in(0,1)$ such that for every $\mathrm{x}, \mathrm{y} \in \mathrm{X}$ and $\mathrm{t}>$

$\int_{0}^{F}\left(\begin{array}{c}M^{2}(P x, Q y, \theta, k t), M^{2}(A x, S y, \theta, t), M^{2}(P x, A x, \theta, t), \\ M^{2}(Q y, S y, \theta, t), M^{2}(P x, S y, \theta, t), M^{2}(A x, Q y, \theta, t)\end{array}\right) \xi(v) d v \geq 1$

Where $\xi:[0,+\infty] \rightarrow[0,+\infty]$ is a lebgesgue integrable mapping which is summable on each compact subset of $[0,+\infty]$, non negative, and such that, $\forall \varepsilon>0$, $\int_{0}^{\varepsilon} \xi(\mathrm{v}) \mathrm{dv}>0$. Then A, S,P and Q have a unique common fixed point in $\mathrm{X}$.

Proof If we take $\mathrm{B}=\mathrm{T}=\mathrm{I}$ ( identity mapping) in Theorem 3.1 then we get the result.

\section{REFERENCES}

[1] Branciari. A. A fixed point theorem for mappings satisfying a general contractive condition of integral type. Int. J. Math. Math. Sci. 29 (2002), no. 9, 531-536.

[2] Cho Y.J., Fixed points in fuzzy metric spaces, J. Fuzzy Math. 5 (4) (1997) 949-962.

[3] Grabiec M. , Fixed points in fuzzy metric spaces, Fuzzy Sets and Systems 27 (1988) 385-389. http://dx.doi.org/10.1016/0165-0114(88)90064-4.

[4] Mishra, S. N.: Common fixed points of compatible mappings in PM -spaces, Math. Japon., 36(2) (1991), $283-289$.

[5] Vasuki R.,A common fixed point theorem in a fuzzy metric space, Fuzzy Sets and Systems 97 (1998) 395397.http://dx.doi.org/101016/S0165-0114(96)00342-9. 